\title{
Téoros
}

Revue de recherche en tourisme

\section{Le tourisme national au Maroc}

Opportunités et limites de développement

\section{Brahim Moudoud et Abdelkrim Ezaïdi}

Volume 24, numéro 1, printemps 2005

Maroc, réalités et défis

URI : https://id.erudit.org/iderudit/1071106ar

DOI : https://doi.org/10.7202/1071106ar

Aller au sommaire du numéro

Éditeur(s)

Université du Québec à Montréal

ISSN

0712-8657 (imprimé)

1923-2705 (numérique)

Découvrir la revue

Citer cet article

Moudoud, B. \& Ezaïdi, A. (2005). Le tourisme national au Maroc : opportunités et limites de développement. Téoros, 24(1), 25-30.

https://doi.org/10.7202/1071106ar d'utilisation que vous pouvez consulter en ligne.

https://apropos.erudit.org/fr/usagers/politique-dutilisation/ 


\section{Le tourisme national au Maroc Opportunités et limites de développement}

\section{Brahim Moudoud et Abdelkrim Ezaïdi}

Longtemps considéré comme le parent pauvre du tourisme marocain, le tourisme national ne fait parler de lui qu'en période de crise du tourisme international. II intervient comme élément essentiel des plans anticrise et s'est vu accorder, durant les deux dernières années (2003-2004), une importance relative, aussi bien de la part des responsables du tourisme que des opérateurs touristiques privés. Quant aux mesures statistiques fiables, notons qu'une part des nuitées touristiques nationales passées chez les parents, les amis ou dans les logements occasionnels privés échappe à l'enregistrement statistique aussi bien des services de sécurité que de ceux du ministère du Tourisme. De plus, les données statistiques des établissements d'hébergement classés peuvent être entachées d'erreurs et leurs déclarations peuvent être faussées (Moudoud, 2000 : 234).

En effet, le Maroc, pays en développement, a fondé sa politique touristique sur la base d'un tourisme récepteur et ce, depuis l'instauration de celle-ci au cours du plan triennal de développement économique et social 1965-1967 ${ }^{1}$. Cette tendance s'est affirmée depuis l'époque du protectorat français, pour permettre aux seuls fonctionnaires de l'administration civile et militaire locale et ressortissants français, en visite au Maroc, de passer des vacances, en encourageant la réalisation de relais, de gîtes d'étape et de centres d'estivage (Bakkali, 1997 : 74).

Le tourisme national, dénommé encore tourisme domestique, intérieur ou interne, constitue un apport considérable au développement économique régional par la répartition d'une part des richesses nationales. II per-

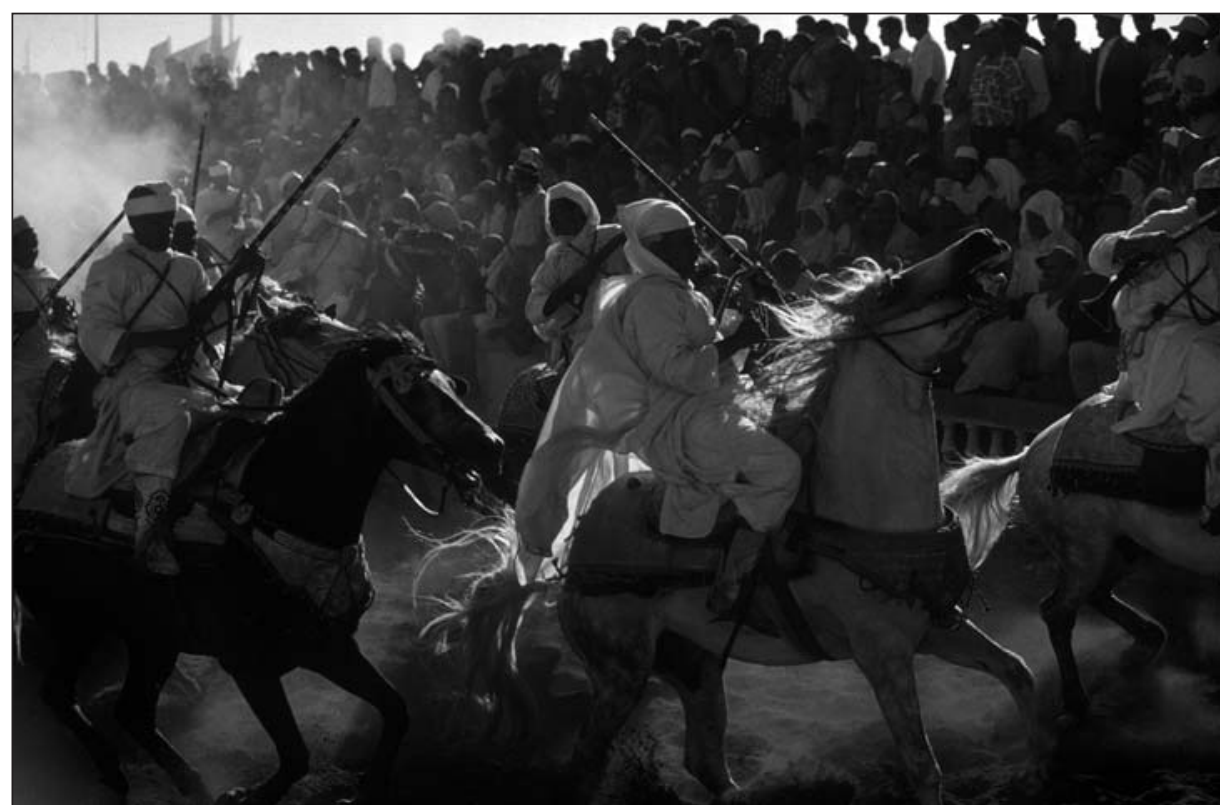

Fantasia dans la ville d'El Jadida (Maroc).

Photo : tourisme-marocain.com

met, outre l'atténuation des effets de la saisonnalité du tourisme international sur la rentabilité des établissements d'hébergement, en particulier, et sur celle des entreprises touristiques, en général, le maintien des emplois et des effets induits sur les transports divers et les autres entreprises de services : cafés, restaurants...

La reprise du tourisme international relègue le tourisme domestique au second rang. C'est dire que le tourisme a toujours été assimilé, dans l'esprit des Marocains, voire dans celui de certains responsables et professionnels, à une activité, ou plutôt à une pratique réservée aux touristes étrangers, tout en marginalisant ainsi les populations locales. Cet esprit est nourri d'une culture traditionnelle en conflit avec un système de développement économique exogène ; comme l'explique Mimoun Hillali (1999:
35-36), "au niveau national, elles [les sociétés traditionnelles] passent pour être des "laissées pour compte [...] [et] au niveau international, les nations où subsistent des sociétés traditionnelles occupent une place périphérique et sont qualifiées de sous-développées!»

\section{Le tourisme national et les crises du tourisme international}

Le tourisme national représente, à notre avis, une demande à potentiel sûr, quelles que soient les conditions internes du pays. II peut également servir d'alternative en cas de régression de la fréquentation touristique internationale ou un outil compensatoire du tourisme international en période de basse saison. 


\section{Le tourisme intérieur: un créneau difficile à cerner}

Au Maroc, les migrations de loisirs ont pris de l'ampleur depuis le milieu des années 1980, par l'adoption de certains comportements du touriste étranger par les nationaux. Le tourisme national pourrait être estimé à $40 \%$ du volume des nuitées touristiques, si I'on considère la fréquentation des établissements d'hébergement non homologués (Berriane : 2002). En effet, d'autres formes de déplacements habituels en vacances utilisent les hébergements chez la famille, les amis et les particuliers (logement chez l'habitant, location de maison et d'appartement privé et sous la tente ${ }^{2}$ ). Ils représentent un volume de visiteurs nationaux difficilement quantifié, qui peut à notre avis se situer aux environs de $50 \%$ et même davantage (Moudoud, 2000 : 254).

En 1989, le colloque franco-marocain sur le tourisme social a été une occasion pour annoncer des mesures en faveur du tourisme national. Une circulaire du ministre du Tourisme a été adressée aux établissements d'hébergement pour accorder une réduction de $25 \%$ sur les prestations d'hébergement à partir de la deuxième nuitée pour les Marocains et les résidents étrangers au Maroc et de $50 \%$ pour les militaires et les anciens combattants. Cette mesure, peu appliquée par les hôteliers et peu diffusée auprès des citoyens, bénéficie à une catégorie de personnes et ne concerne pas les établissements non classés. Elle répond, à notre avis, à une baisse de la fréquentation touristique internationale due aux effets de la pratique du «surbooking " par les hôteliers marocains pendant la saison printanière des années 1987 et $1988^{3}$.

\section{Le tourisme national : solution partielle ou leurre économique pour les crises touristiques internationales}

À la suite des effets de la première crise du Golfe (1990-1991), le tourisme des nationaux au Maroc a contribué - à hauteur de 28,4 \% des nuitées touristiques - au moment où les nuitées du tourisme international ont chuté de $36,5 \%$. Ces résultats sont le fruit de campagnes promotionnelles, souvent isolées, en faveur des nationaux, lancées par les professionnels du tourisme, sous forme de forfaits à prix réduits pour les familles marocaines, pour sauver les saisons touris- tiques hivernale, printanière et estivale et ainsi rentabiliser les investissements hôteliers et ce, bien que cette clientèle ne soit jugée ni meilleure, ni pire que celle des autres pays ${ }^{4}$. II s'agit généralement d'initiatives privées des hôteliers, en l'absence des organes institutionnels et associatifs concernés.

Les villes les plus touchées par cette crise sont Agadir et Marrakech. Elles ont vu la fréquentation de leurs établissements d'hébergement diminuer au point d'atteindre un seuil très bas. Durant les années 1990, à cause de la crise du Golfe et de l'attentat de I'hôtel Atlas Asni à Marrakech en avril 1994, le discours administratif, politique et professionnel n'a cessé de parler du tourisme des nationaux et de la nécessité de son développement.

En termes de stratégie politique, la réaction du gouvernement marocain arrive tardivement en 2000, alors que le tourisme marocain était en train de récupérer timidement les parts perdues du marché, par la commande d'une étude sur le tourisme intérieur, en juillet 2000, étude qui s'est achevée vers la fin du mois de mai $2002^{5}$. Cette dernière, quelles que soient ses conclusions, servira, après la grande crise du 11 septembre et le coup dur contre la libre circulation et le déplacement de personnes à travers le monde, à lancer l'opération Kounouz Biladi 6 à partir du printemps 2003.

\section{Tableau 1}

Variation annuelle des arrivées et des nuitées touristiques du tourisme national dans les hôtels classés, de 1994 à 2003

\begin{tabular}{l|c|c|c|c|c|c|c|c|c|c}
\hline & $\mathbf{1 9 9 4}$ & $\mathbf{1 9 9 5}$ & $\mathbf{1 9 9 6}$ & $\mathbf{1 9 9 7}$ & $\mathbf{1 9 9 8}$ & $\mathbf{1 9 9 9}$ & $\mathbf{2 0 0 0}$ & $\mathbf{2 0 0 1}$ & $\mathbf{2 0 0 2}$ & $\mathbf{2 0 0 3}$ \\
\hline Arrivées & $-2,8$ & 1,3 & $-0,8$ & 5,5 & 0,7 & $-2,2$ & 3,6 & 3,4 & 5 & 6,6 \\
Nuitées & $-3,2$ & $-2,2$ & 1,2 & 3,5 & 0,7 & $-2,7$ & 2,4 & 5,7 & 2,2 & 8,3 \\
\hline
\end{tabular}

Source : Ministère du Tourisme, statistiques annuelles de 1994 à 2003.

Tableau 2

Comparaison des parts annuelles des arrivées et des nuitées touristiques du tourisme national et international dans les hôtels classés, de 1994 à 2003

\begin{tabular}{l|l|l|l|l|l|l|l|l|l|l|c}
\hline & & $\mathbf{1 9 9 4}$ & $\mathbf{1 9 9 5}$ & $\mathbf{1 9 9 6}$ & $\mathbf{1 9 9 7}$ & $\mathbf{1 9 9 8}$ & $\mathbf{1 9 9 9}$ & $\mathbf{2 0 0 0}$ & $\mathbf{2 0 0 1}$ & $\mathbf{2 0 0 2}$ & $\mathbf{2 0 0 3}$ \\
\hline \multirow{2}{*}{ Arrivées } & T. Récepteur & 72,7 & 67,7 & 69 & 70,6 & 72,4 & 75,5 & 75,8 & 73,4 & 69,2 & 66,2 \\
& T.Interne & 27,3 & 32,3 & 31 & 29,4 & 27,6 & 24,5 & 24,2 & 26,6 & 30,8 & 33,8 \\
\multirow{2}{*}{ Nuitées } & T. Récepteur & 80,8 & 78,3 & 78,3 & 79,3 & 81 & 83 & 83,2 & 81,1 & 78,3 & 76,2 \\
& T.Interne & 19,2 & 21,7 & 21,7 & 20,7 & 19 & 17 & 16,8 & 18,9 & 21,7 & 23,8 \\
\hline
\end{tabular}

Source : Ministère du tourisme, statistiques annuelles de 1994 à 2003.

\section{Évolution du tourisme national au Maroc}

\section{L'évolution des arrivées et des nuitées touristiques}

En référence aux statistiques des arrivées et des nuitées touristiques du tourisme des Marocains (Ministère du Tourisme marocain,1985), nous constatons que ce type de tourisme présente une évolution lente de 1985 à 2000, avec des taux moyens d'accroissement annuel, respectivement de 2,0\% et de3,8\%. Durant cette période, la part des nuitées du tourisme national dans établissements d'hébergement classés oscille de $15 \%$ à $20 \%$, avec une nuance me national ont atteint $28,4 \%$ du volume global des nuitées, à cause de la régression des mière guerre du Golfe.

Toutefois, l'analyse de l'évolution du tourispas affecté par les dernières crises du tourisme international, à savoir: les événements du 11 septembre 2001, la guerre contre les Talibans, la deuxième guerre de 'Iraq, les attentats du 16 mai 2003 à asablanca et du 11 mars 2004 à Madrid (tableaux 1 et 3). Son évolution et sa part annuelle dans le tourisme marocain sont inestimables, même si elles présentent une évolution constante (tableau 2). Les nuitées passées dans les hôtels classés ont évolué pour l'année 1991, où les nuitées du tourisme national au Maroc a démontré qu'il n'est 
Arrivées touristiques par marché dans les hôtels classés, de 2000 à 2003

\begin{tabular}{|c|c|c|c|c|c|c|c|c|c|c|c|c|c|c|}
\hline & \multicolumn{2}{|c|}{2000} & \multicolumn{4}{|c|}{2001} & \multicolumn{4}{|c|}{2002} & \multicolumn{4}{|c|}{2003} \\
\hline & Arrivées & Nuitées & Arrivées & $\begin{array}{c}2001 / 2000 \\
(\%)\end{array}$ & Nuitées & $\begin{array}{c}2001 / 2000 \\
(\%)\end{array}$ & Arrivées & $\begin{array}{c}2002 / 2001 \\
(\%)\end{array}$ & Nuitées & $\begin{array}{c}2002 / 2001 \\
(\%)\end{array}$ & Arrivées & $\begin{array}{c}2003 / 2002 \\
(\%)\end{array}$ & $\begin{array}{c}\text { Nuitées } \\
(\%)\end{array}$ & $2003 / 2002$ \\
\hline \multicolumn{15}{|l|}{ Pays } \\
\hline France & 1453581 & 4691544 & 1370469 & $-5,72$ & 4436713 & $-5,43$ & 1202764 & $-12,24$ & 4152369 & $-6,41$ & 1206656 & 0,32 & 4329870 & 4,27 \\
\hline Allemagne & 407570 & 1833759 & 354436 & $-13,04$ & 1563578 & $-14,73$ & 234322 & $-33,89$ & 1089132 & $-30,34$ & 149131 & $-36,36$ & 761604 & $-30,07$ \\
\hline Angleterre & 135859 & 544767 & 145700 & 7,24 & 606882 & 11,40 & 129659 & $-11,01$ & 485281 & $-20,04$ & 112407 & $-13,31$ & 450654 & $-7,14$ \\
\hline Italie & 249701 & 750212 & 227850 & $-8,75$ & 663287 & $-11,59$ & 197718 & $-13,22$ & 551389 & $-16,87$ & 153681 & $-22,27$ & 439014 & $-20,38$ \\
\hline Belgique & 87238 & 452420 & 84851 & $-2,74$ & 435533 & $-3,73$ & 77446 & $-8,73$ & 386412 & $-11,28$ & 69580 & $-10,16$ & 356957 & $-7,62$ \\
\hline Espagne & 275324 & 589982 & 227527 & $-17,36$ & 468163 & $-20,65$ & 169656 & $-25,43$ & 344587 & $-26,40$ & 172625 & 1,75 & 354495 & 2,88 \\
\hline U.S.A & 149750 & 323569 & 116744 & $-22,04$ & 257365 & $-20,46$ & 80974 & $-30,64$ & 185068 & $-28,09$ & 58123 & $-28,22$ & 133333 & $-27,95$ \\
\hline Japon & 67157 & 106778 & 43079 & $-35,85$ & 75664 & $-29,14$ & 38723 & $-10,11$ & 64729 & $-14,45$ & 36417 & $-5,96$ & 61275 & $-5,34$ \\
\hline Canada & 26985 & 77840 & 22054 & $-18,27$ & 59097 & $-24,08$ & 16330 & $-25,95$ & 42780 & $-27,61$ & 16145 & $-1,13$ & 43293 & 1,20 \\
\hline \multicolumn{15}{|l|}{ Régions } \\
\hline Moyen Orient & 74167 & 284453 & 71925 & $-3,02$ & 283223 & $-0,43$ & 73870 & 2,70 & 289250 & 2,13 & 68555 & $-7,20$ & 266923 & $-7,72$ \\
\hline Scandinavie & 65916 & 449989 & 56584 & $-14,16$ & 371698 & $-17,40$ & 30149 & $-46,72$ & 179303 & $-51,76$ & 36325 & 20,48 & 235291 & 31,23 \\
\hline Maghreb & 32819 & 94934 & 35850 & 9,24 & 100446 & 5,81 & 35312 & $-1,50$ & 105483 & 5,01 & 35806 & 1,40 & 111573 & 5,77 \\
\hline Afrique & 23305 & 57566 & 25262 & 8,40 & 63912 & 11,02 & 28250 & 11,83 & 78735 & 23,19 & 34548 & 22,29 & 78300 & $-0,55$ \\
\hline Autres & 338355 & 1009948 & 300968 & $-11,05$ & 907563 & $-10,14$ & 317334 & 5,44 & 911479 & 0,43 & $296497^{7}$ & $-6,57$ & $892711^{8}$ & $-2,06$ \\
\hline T. Récepteur & 3387727 & 11267761 & 3083299 & $-8,99$ & 10293124 & $-8,65$ & 2632507 & $-14,62$ & 8865997 & $-13,86$ & 2446496 & $-7,07$ & 8515293 & $-3,96$ \\
\hline T. Interne & 1079512 & 2271806 & 1116615 & 3,44 & 2402104 & 5,74 & 1172843 & 5,04 & 2454885 & 2,20 & 1250454 & 6,62 & 2657826 & 8,27 \\
\hline Total & 4467239 & 13539567 & 4199914 & \begin{tabular}{|l|}
$-5,98$ \\
\end{tabular} & 12695228 & $-6,24$ & 3805350 & $-9,39$ & 11320882 & $-10,83$ & 3696950 & $-2,85$ & 11173119 & $-1,31$ \\
\hline
\end{tabular}

Source: Nos calculs, à partir des données du ministère du Tourisme marocain.

légèrement de 1992 à 2003, en passant seulement de 2334 millions à 2657 millions de nuitées.

Par contre, les taux moyens d'accroissement annuel des arrivées et des nuitées touristiques du tourisme national se sont sensiblement améliorés pour la période allant de 2000 à 2003 ; ils sont respectivement de $4,65 \%$ et de $5,4 \%$, avec un ralentissement de la croissance des nuitées en 2002 de 2,2\% (tableau 3).

La part des arrivées du tourisme intérieur dans les hôtels classés en 2001 était de 26,6 \%, avec une augmentation de 3,4 \% par rapport à 2000. Quant aux années 2002 et 2003, au chapitre des arrivées enregistrées dans les hôtels classés, leur part a franchi pour la première fois la barre de $30 \%$ pour atteindre, respectivement, 33,8 \% et 23,8 \% du volume global de l'activité touristique en 2003 (tableau 2) et les taux d'accroissement annuel des arrivées et des nuitées étaient respectivement de $5 \%, 6,6 \%$ et de 2,2 \%, 8,3\%.
En ce qui concerne la part de chaque marché, le tableau 3 renseigne sur l'importance des arrivées du marché national par rapport aux autres marchés touristiques. En 2003, il a détrôné le marché français, considéré comme marché touristique traditionnel pour le Maroc, en représentant 33,8\% du total des parts des arrivées des marchés touristiques, suivi en deuxième place par le marché français avec 32,6\%. Le marché italien occupe la troisième place avec seulement 6,3\% (tableaux 3 et 4). En termes de chiffres absolus, les arrivées des nationaux dans les hôtels classés en 2003 se chiffrent à 1250454 contre 1206656 touristes français (tableau 3).

Par contre, en termes de nuitées, les Français sont en tête avec 4329293 contre 2657826 nuitées pour les nationaux, soit, en termes de part de chaque nationalité : $38,8 \%$ pour les premiers et 23,8\% pour les seconds (tableau 3), ce qui explique la courte durée des séjours des touristes nationaux.
Par ailleurs, les chiffres des autres nationalités montrent les fortes régressions des NordAméricains (USA et Canada), des Italiens, des Scandinaves et des Japonais (tableau 3).

Les variations annuelles par marché montrent les effets des évènements du 11 septembre 2001 et de la deuxième guerre du Golfe. Seul le tourisme interne a résisté à la régression annuelle sur les trois dernières années. Sa part a augmenté, quand celle du tourisme récepteur a régressé.

Les variations des arrivées et des nuitées du tourisme national présentent une corrélation de 1995 à 2000, avec une baisse importante de 1997 à 1999. Par contre, de 2000 à 2003, les variations semblent incohérentes avec de fortes variations en 2001 et en 2003.

Ces résultats récents du tourisme national sont, à notre avis, le fruit de l'impact de l'opération Kounouz Biladi, lancée au printemps 2003. Ils se traduisent, à notre avis, par une fréquentation massive des établissements hôteliers classés par les nationaux. 
Toutefois, les estimations de l'étude de la stratégie d'aménagement touristique (Ministère du Tourisme, 1996) élaborée en 1996 n'ont pas encore été atteintes (tableaux 4 et 5).

\section{Prévisions et réalisations de l'étude de la stratégie d'aménagement touristique}

L'étude de la stratégie d'aménagement touristique - dans son scénario retenu (Ministère du Tourisme, 1996: 52 et 58) - a estimé la demande interne à 18 millions de nuitées en 2005 et à 23 millions en 2010, toutes catégories d'hébergement confondues.

Quant à l'hôtellerie classée, cette étude a prévu un accroissement de sa part de la demande interne d'environ 2,2 \%, sur la base de l'évolution des années précédentes. Nous avons adopté le même pourcentage pour le calcul des estimations des années 2000, 2005 et 2010.

La comparaison des données relatives aux estimations de 1996 et aux résultats de l'année 2000 (tableaux 4 et 5) montre que ces résultats sont inférieurs aux estimations de l'étude. L'écart dégagé des arrivées du tourisme international dans les hôtels classés est de 29,42 \%, au moment où celui-ci se porte bien en raison du climat politique favorable et de la relance des migrations de loisirs sur toutes les destinations touristiques mondiales. La situation de 1996 à 2000 n'a par ailleurs présenté aucune perturbation du flux touristique international.

Quant au tourisme national, l'estimation des arrivées pour l'année 2000 est inférieure de $8,4 \%$ par rapport aux projections faites

\section{Tableau 4}

Écarts dégagés des arrivées et des nuitées touristiques dans les hôtels classés en 2000

\begin{tabular}{|c|c|c|c|}
\hline & 1993 & 2000 & Écart \\
\hline \multicolumn{4}{|l|}{ Arrivées } \\
\hline T. Récepteur & 2723204 & 3387727 & $-29,42 \%$ \\
\hline T. Interne & 1011953 & 1079512 & $-8,39 \%$ \\
\hline \multicolumn{4}{|l|}{ Nuitées } \\
\hline T. Récepteur & 10003000 & 11267761 & $-14,24 \%$ \\
\hline T. Interne & 2285000 & 2271806 & $-14,56 \%$ \\
\hline \multicolumn{4}{|c|}{$\begin{array}{l}\text { Source: Nos calculs, à partir des données du ministère du } \\
\text { Tourisme marocain pour les années } 1993 \text { et } 2000 \text {. }\end{array}$} \\
\hline
\end{tabular}

\section{Tableau 5}

Estimation des arrivées et des nuitées touristiques dans les hôtels classés, de 2000 à 2010

\begin{tabular}{|c|c|c|c|c|c|}
\hline & & $1993^{9}$ & 2000 & 2005 & 2010 \\
\hline \multirow[t]{2}{*}{ Arrivées } & T. récepteur ${ }^{10}$ & 2723204 & 4800000 & 6200000 & 7000000 \\
\hline & T.Interne & 1011953 & 1178465 & 1313927 & 1464960 \\
\hline \multirow[t]{2}{*}{ Nuitées } & T. Récepteur & 10003000 & 13139000 & 17431000 & 23147000 \\
\hline & T. Interne & 2285000 & 2659000 & 2965000 & 3304000 \\
\hline
\end{tabular}

Source: Nos calculs, à partir des données du ministère du Tourisme marocain (1996).

en 1996 et demeure ainsi proche de la réalité. Par contre, les nuitées réalisées ont accusé un écart de $-14 \%$, tant pour le tourisme international que pour le tourisme national.

\section{La promotion du tourisme national}

Le début du millénaire s'est entaché de beaucoup d'événements perturbateurs du mouvement touristique international. La tendance est actuellement vers une relance de cette activité, mais des problèmes majeurs subsistent, notamment les restrictions douanières et les contrôles policiers, qui causent des désagréments aux touristes. Les risques liés au transport aérien freinent davantage les déplacements de personnes au niveau international.

\section{Le tourisme national et la vision 2010}

Si le Contrat-Programme 2001-2010 qui lie le Gouvernement et la Fédération du Tourisme de la Confédération Générale des Entreprises du Maroc a omis le tourisme intérieur, l'accord d'application de l'accord-cadre 2001-2010 ${ }^{11}$ lui a réservé un intérêt qui consiste en l'organisation de fins de semaine et de packages par le biais d'opérations promotionnelles, tout en impliquant les secteurs clés capables de relancer ce type ce tourisme: les compagnies de transport aérien, ferroviaire et terrestre, les fédérations nationales des agents de voyages et de l'industrie hôtelière. Cette vision recommande un intérêt particulier au développement de ce créneau du tourisme marocain, sans trop préciser les moyens d'y parvenir. Les assises du tourisme nationales annuelles, depuis janvier 2001, mettent l'accent beaucoup plus sur le tourisme international que sur le tourisme domestique.

\section{La mesure de la demande du tourisme national}

L'étude élaborée de juillet 2000 à mai 2002 (Ministère du Tourisme, 2002), qui a porté sur 6000 Marocains, dégage que les migrations de loisirs internes produisent 7,5 millions de départs en voyages, dont $75 \%$ pour des vacances, $19 \%$ pour d'autres loisirs et $6 \%$ pour affaires. Sur les 7,5 millions, il y a $89 \%$ de longs séjours, dont $94 \%$ sans réservation à l'avance. En outre, 88 \% sont effectués en voiture ou en autocar, car la couverture du réseau de chemin de fer est limitée sur le territoire national au nord (Tanger), à l'est (Fès et Oujda) et s'arrête au sud à Marrakech. Ainsi, les nuitées produites par le tourisme national s'élèvent, d'après cette enquête, à 117 millions de nuitées, dont $80 \%$ pour les vacances, $16 \%$ pour les autres loisirs et $4 \%$ seulement pour le tourisme d'affaires. La moyenne de nuitées des différents voyages est respectivement de $16,4,12,7$ et 11,8 nuitées.

L'étude répertorie trois types d'hébergement:

$75 \%$ - hébergement gratuit ;

$14 \%$ - autres hébergements payants ;

$11 \%$ - hébergement hôtelier classé.

Ces chiffres donnent matière à réflexion et montrent l'importance du volume des déplacements des Marocains et la nature de la consommation des hébergements par cette demande.

\section{Kounouz Biladi : un produit pour la relance du tourisme national ou la semence de la tradition touristique dans la vie du Marocain?}

La campagne Kounouz Biladi est unique en son genre depuis l'instauration de la politique touristique nationale. Loin des mesures d'encouragement fiscal, la baisse des salaires à $50 \%$ pour éviter les licenciements dans le domaine de l'hôtellerie, comme c'était le cas lors de la première crise du Golfe, 
et la baisse des prix des prestations touristiques par les professionnels, l'Office national du Tourisme - en collaboration avec ces derniers - a eu recours à une opération baptisée "Kounouz Biladi ». La première campagne du printemps 2003 vient après la persistance des effets des événements du 11 septembre 2001, le taux d'occupation des hôtels de Marrakech n'a pas dépassé $46 \%$ au mois de mars contre $48,3 \%$ au mois de février 2003. Agadir chiffre des taux encore plus bas : -2,69\% en février, 19,59\% en mars et $-17,87 \%$ en avril 2003.

\section{Degré d'adhésion de la profession à la campagne Kounouz Biladi}

La première opération de Kounouz Biladi a eu lieu du 14 avril au 18 mai 2003, sur une durée d'un mois seulement, qui coïncide en réalité avec la haute saison printanière, suivie d'une campagne timide à l'été $2003^{12}$ et de deux autres campagnes au printemps et à l'été 2004. Les taux de participation à cette campagne des établissements d'hébergement et des agences de voyages ont été, au printemps 2003, respectivement de $16 \%^{13}$ des hôtels classés et de $13 \%{ }^{14}$ des agences de voyages mères. Le taux de participation au printemps 2004 a été à la baisse de $1 \%$ dans les deux cas. C'est à l'été 2004 que l'on constate une hausse du taux de participation à la campagne Kounouz Biladi. Pour les hôtels classés, la hausse est légère et porte le taux à $18 \%$. Cependant, c'est au niveau des agences de voyages mères que le taux de participation augmente considérablement avec un taux à $49 \%{ }^{15}$.

\section{Les limites de développement du tourisme national}

II existe un certain nombre de facteurs qui limitent le développement du tourisme intérieur. Nous nous contentons de soulever certains éléments relatifs aux facteurs socioéconomiques, réglementaires, infrastructurels, organisationnels et professionnels. La promotion du tourisme national passe par une volonté politique qui doit certes être accompagnée de mesures concrètes, susceptibles de la soutenir. Les secteurs public et privé sont appelés à s'impliquer davantage dans la planification d'un programme de réalisations d'infrastructures d'accueil nécessaires pour le tourisme social, en tenant compte des contraintes sociales (familles, familles nombreuses) économiques (revenus) et environnementales (capacité de charge).

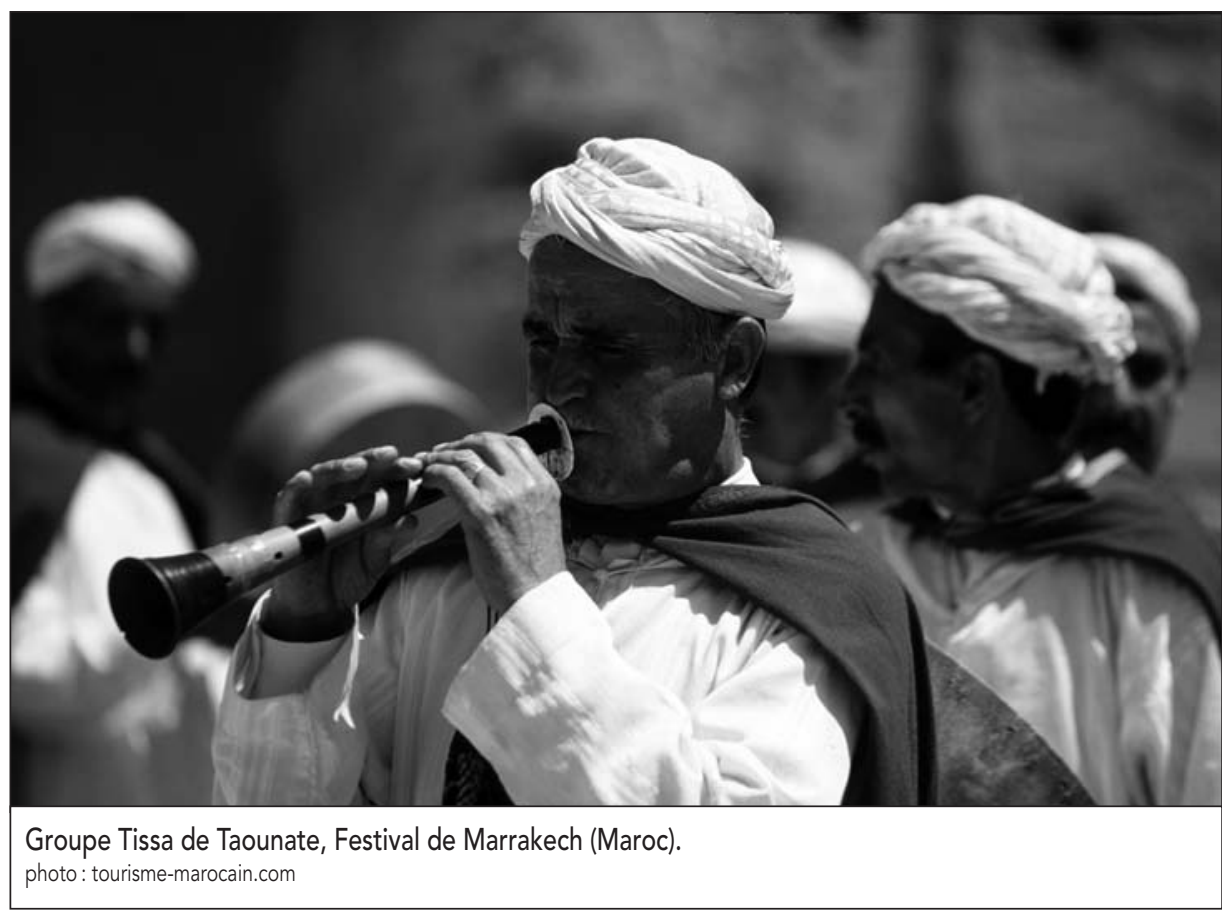

La capacité d'hébergement à caractère social existante doit être exploitée au maximum, avec une répartition équitable et équilibrée, pour en faire bénéficier les fonctionnaires et les employés à revenu faible et les couches sociales les plus démunies. À notre avis, la démocratisation de l'accès au tourisme pourrait inculquer une culture de voyages organisés et réguliers chez les Marocains et ce, en généralisant les droits aux réductions, au lieu qu'elles soient réservées à une élite : parlementaires, militaires et anciens combattants (50\%). Du point de vue réglementaire, les textes de classement sont appelés à être révisés dans le sens d'assurer les hébergements adéquats aux familles marocaines.

Quant aux infrastructures routières actuelles, elles ne répondent pas à la demande des départs en vacances. Les risques d'accidents, dus à l'état des routes et aux excès de vitesse, rebutent un grand nombre de personnes à prendre ces voies. Le réseau ferroviaire, qui présente des déplacements, généralement sans risques, reste limité et ne dessert que les trois axes déjà cités. Les prix des dessertes aériennes internes sont prohibitifs pour les classes moyennes. Les quelques réductions pratiquées ne sont pas généralisées. Sur le plan organisationnel, le rôle des offices du tourisme (Délégation du tourisme) et des syndicats d'initiatives ne répond pas aux besoins et aux attentes des nationaux. II est même enraciné dans les esprits que ces organismes sont réservés à servir le touriste étranger. L'importance de ce créneau incite la tenue de statistiques des arrivées et des nuitées des hôtels non classés et des autres types d'hébergement privé, qui ne sont pas considérés dans les rapports de certaines délégations régionales du tourisme.

Du point de vue professionnel, les agences de voyage nationales s'orientent vers le tourisme international, qui draine une plus value plus importante que celle du tourisme national. Elles sont appelées à investir davantage dans le tourisme national et à faire des efforts pour le hisser au rang qu'il mérite par la diversification du produit touristique national (Ezaïdi, 2001 : 66), d'autant plus que d'autres prestataires de services sont appelés à revoir leur comportement vis-à-vis du client national : les restaurants, les cafés, les Bazars ${ }^{16}$, les taxis..., en évitant de manifester le désintérêt pour la clientèle nationale en haute saison.

\section{Conclusion}

Les migrations de loisirs et les déplacements des nationaux occupent une large frange de la population marocaine. Ils contribuent à la dynamisation des économies locales, notamment dans les stations touristiques balnéaires, culturelles, religieuses et montagnardes. Or, la quantification et l'encadrement restent difficiles. Les responsables manifestent à chaque occasion le souhait de voir se développer un tourisme national, ce 
qui peut épargner les doléances et les pressions des promoteurs privés. Mais, en même temps, on constate que ces derniers ne réagissent pas concrètement ou, plutôt, ils réagissent superficiellement par l'encouragement de campagnes promotionnelles, à effet relativement faible (Kounouz Biladi). Ainsi, ils attendent que les revenus augmentent, que les villes ne supportent plus leurs habitants, que les comportements des citadins marocains changent pour devenir comme ceux des occidentaux, que les enfants exercent des pressions sur les parents et que l'étau des visas se resserre davantage, pour déclencher le mouvement de déplacements des nationaux à l'intérieur du pays.

Afin de participer à combler les vides de la basse saison et à accroître le volume des départs, le tourisme national nécessite des infrastructures d'accueil adéquates et adaptées à la structure familiale marocaine, ainsi que l'encouragement aux départs hors des saisons des vacances scolaires (printemps et été). Le rôle de l'État, notamment les collectivités locales, est de conserver et de gérer à bon escient le patrimoine touristique naturel et d'assurer son exploitation, comme domaine public de l'État, par tous les visiteurs sans exception, qu'ils soient nationaux ou étrangers. La pseudo-privatisation de certaines plages du nord du pays n'est qu'une entrave au développement du tourisme national.

Brahim Moudoud est enseignant-chercheur au Département de géographie de l'Université Ibn Zohr, Faculté des lettres et des sciences humaines.

Abdelkrim Ezaïdi est enseignant-chercheur au département de Géologie, Université Ibn Zohr Faculté des Sciences.

\section{Notes}

1 La part du budget du secteur du tourisme dans ce plan est de 6,4\%, contre 1,4\% lors du plan quinquennal 1960-1964.

2 II s'agit de nuitées passées sous tentes à l'occasion de moussems (rassemblements populaires à caractère religieux, commercial et tribal), de festivals ou sur les plages. Nombreuses sont les manifestations de ce genre en 2004

3 La pratique de «surbooking " ou «la vacance de l'offre" consiste à vendre un nombre de chambres supérieur à la capa- cité réelle d'un établissement d'hébergement. Elle est pratiqué également par les agents de voyages.

4 Vie touristique africaine, "Le tourisme national: une clientèle ni meilleure, ni moins bonne que celle des autres pays ", bimensuel touristique, $\mathrm{n}^{\circ} 430$ du 5 septembre 1991, p. 18.

5 Étude réalisée par le consortium : le cabinet T.H.R. (Barcelone) et l'Institut Interstrategic (Casablanca), en collaboration avec IPK international (Munich)

6 Traduite sous la dénomination «Trésors de mon pays ", cette opération est une campagne de promotion du tourisme national par l'offre de packages dans les hôtels classés du Royaume en 2003 et 2004.

7 Pour l'année 2003, les Marocains Résidants à l'Étranger (MRE) représentent 14586 touristes; les ressortissants autrichiens se chiffrent à 18555 ; les touristes en provenance de la Communauté d'États indépendants (C.E.I.: anciennes républiques de I'Union soviétique) sont au nombre de 4522; les touristes en provenance du Portugal sont au nombre de 30789 ; ceux de la Hollande 46376 et ceux de la Suisse 38 269. Ils sont tous groupés dans «Autres».

8 Idem pour les nuitées des six nationalités incluses dans «Autres »: M.R.E 25195 nuitées, Autriche 40432 nuitées, C.E.I. 26839 nuitées, Portugal 91840 nuitées, Hollande 136800 nuitées et Suisse 129539 nuitées.

9 Chiffres représentant la base de l'étude de la stratégie d'aménagement touristique en 1995 et 1996.

10 A tenu compte du tourisme des Algériens (tourismes transfrontalier et de transit) et des MRE.

11 Document d'accord entre le Gouvernement et la Fédération du Tourisme de la Confédération Générale des Entreprises du Maroc (FT-CGEM). II détaille les éléments contenus dans l'accord-cadre et préconise les actions à entreprendre pour chacun d'eux.

12 À cause des attentats du 16 mai 2003 à Casablanca

13 À remarquer qu'il y avait, au 31 décembre 2003, 632 établissements hôteliers classés. Ils servent de base de calcul pour l'année 2004.

14 Les agences de voyages mères du Maroc sont au nombre de 512, à l'exception des succursales, au 31 décembre 2003. Le total des agences de voyages du Maroc, y compris les succursales, est de 715 agences.

15 Office national marocain du Tourisme, publications pour la campagne Kounouz Biladi (2003 et 2004) et Ministère du Tourisme, Capacité d'hébergement et nombre d'agent de voyages au 31 décembre 2003.
16 Ce sont les commerces des produits d'artisanat. Le mot Bazariste désigne le commerçant de ces produits.

\section{Bibliographie}

Bakkali, Mohamed (1997), Droit du tourisme au Maroc : étude analytique et critique, Tome 1 : Organisation du phénomène touristique, Rabat (édition arabe).

Berriane, Mohamed (1978), "L'espace touristique marocain ", Thèse de $3^{e}$ cycle, Université de Tours.

Berriane, Mohamed (1993), «Le Tourisme des nationaux au Maroc : une nouvelle approche du tourisme dans les pays en voie de développement », Annales de Géographie, n 570, p. 131-161

Berriane, Mohamed (1994), «Tourisme national et Tourisme international au Maroc : une réelle complémentarité ", Téoros, vol. 13, n 2, été, p. 29-33.

Berriane, Mohamed (2002), Les nouvelles tendances du développement du tourisme au Maroc, Actes du $13^{\mathrm{e}}$ festival international de Géographie, Religion et géographie, Saint-Dié des Vosges.

Ezaïdi, Abdelkrim (2001), Importance de la biodiversité et de la géodiversité dans le contexte touristique régional et national, Actes du colloque international Le Tourisme des jeunes, Université Cadi Ayyad, Marrakech, p. 66-68.

Hillali, Mimoun (1999), Tourisme et patrimoine: duo ou duel socioculturel?, Actes du colloque Le Tourisme en question, Série Colloques 11, Meknès, Faculté des Lettres et Sciences humaines de l'Université Moulay Ismail, p. 35-42.

Ministère du Tourisme (1996), Étude de la stratégie d'aménagement touristique: Stratégie de développement et programme d'action, Rabat, Rapport final, Urbaplan, avril, 219 p.

Ministère du Tourisme marocain (1985-2003), Le secteur touristique: Statistiques, Direction de la planification et de la coordination de la promotion, Rabat, de 1985 à 2003.

Ministère du Tourisme marocain (2002), Étude sur le tourisme intérieur, Direction de la planification et de la coordination de la promotion, Rabat, 59 p.

Moudoud, Brahim (2000), «Production et gestion du tourisme de montagne au Maroc », thèse de doctorat, Université Joseph Fourier, Grenoble 1. 